\title{
Increase the rate of plasma-assisted synthesis of silver nanoparticles through additives
}

\author{
Zhai Limin ${ }^{1}$, Lu Ye $^{2}$, Chen Dong ${ }^{3}$, Chen Xinyi ${ }^{3}$, Liu Linsheng ${ }^{4}$ and Li Chuanqi ${ }^{5}$ \\ ${ }^{1}$ Zhai Limin, College of Electronic Engineering, Guangxi Normal University, China \\ ${ }^{2} \mathrm{Lu}$ Ye, College of Electronic Engineering, Guangxi Normal University, China \\ ${ }^{3}$ Chen Dong, College of Physics and Electronics, Nanning Normal University, China \\ ${ }^{3}$ Chen Xinyi, College of Electronic Engineering, Guangxi Normal University, China \\ ${ }^{4}$ Liu Linsheng, College of Electronic Engineering, Guangxi Normal University, China \\ ${ }^{5} \mathrm{Li}$ Chuanqi, College of Physics and Electronics, Nanning Normal University, China
}

\begin{abstract}
The plasma-assisted method was used to synthesize silver nanoparticles, and the growth process of silver nanoparticles (Ag-NPs) was monitored in real time by localized surface plasmon resonance (LSPR) absorption spectroscopy. The effect of additives on the synthesis of Ag-NPs was verified. It is found that the addition of isopropanol and glucose can increase the plasmon resonance absorption intensity of the reaction solution, and promote the synthesis of Ag-NPs. In the plasma-assisted method, the additives can effectively improve the synthesis efficiency of Ag-NPs, which has great inspiration for the synthesis of other metal nanoparticles.
\end{abstract}

\section{Introduction}

In recent years, the research and application of nanotechnology has become more and more extensive, especially the noble metal nanomaterials have gradually been applied to the fields of biomedicine [1], environmental health [2], photocatalysis [3] and new energy ${ }^{[4]}$. Among them, silver nanoparticles (Ag-NPs) have antibacterial and bactericidal properties ${ }^{[5]}$, which are particularly prominent in biomedical applications. The current methods for synthesizing Ag-NPs are mostly chemical methods ${ }^{[6]}$, but these methods require the introduction of toxic and harmful reducing agents during the experiments, which will pollute the environment. Plasma assisted method uses part of the reducing substances generated after the discharge plasma contacts the liquid surface to replace the reducing agent added in the chemical method to reduce silver ions ${ }^{[7]}$. This method has the advantage of being more environmentally friendly. And the experimental equipment used is simpler to synthesize metal nanoparticles on a large scale.

So far, there are few researches on the synthesis of silver nanoparticles by plasma electrochemical method at domestic and abroad. Chen Q and others have carried out some related mechanism and application research ${ }^{[8-10]}$. They studied plasma-liquid reaction system by changing the current and adding different active materials. In this paper, a control group experiment was set up. Isopropanol and glucose were added and the concentration was changed. The growth process of Ag-NPs was monitored in real time by localized surface plasmon resonance (LSPR) absorption spectroscopy, so as to discuss the influence of these two additives on the growth rate of Ag-NPs.

\section{Experimental Setup}

When the discharge plasma contacts the liquid surface, reducing and oxidizing substances are generated. ${ }^{[10]}$ The plasma-assisted method for synthesizing Ag-NPs uses these reducing substances (mainly hydrated electrons, atomic $\mathrm{H}$, etc.) in the precursor solution to reduce $\mathrm{Ag}^{+}$to $\mathrm{Ag}^{0}$. The reducing substances and oxidizing substances in the solution coexist, so the reduction reaction and the oxidation reaction in the reaction system proceed simultaneously. The main reductions in the solution are as follows:

$$
\begin{aligned}
& e+\mathrm{H}_{2} \mathrm{O} \rightarrow e_{a q}{ }^{-} \\
& e_{a q}{ }^{-}+\mathrm{Ag}^{+} \longrightarrow A g
\end{aligned}
$$

To prevent the Ag-NPs generated in the solution from agglomerating during the synthesis process, polyvinyl pyrrolidone (PVP) was added to the solution.

The setup used in the experiment is shown in Figure 1. In the experiment, a negative voltage (high voltage DC power supply LAS-20KV-50mA) was applied to the tungsten steel electrode $3 \mathrm{~mm}$ above the liquid surface to excite plasma, and argon passed through the gas flow controller (D08-3F, Qixing Huachuang).Flow rate of the electrode nozzle is controlled at $20 \mathrm{sccm}$. The circulation process of the reaction is as follows: First, a peristaltic pump (RZ1030 box type, Runze fluid CO.Ltd) pumps

"Liu Linsheng: linshengliu@163.com ${ }^{*}$ Li Chuanqi:lcq@gxnu.edu. 


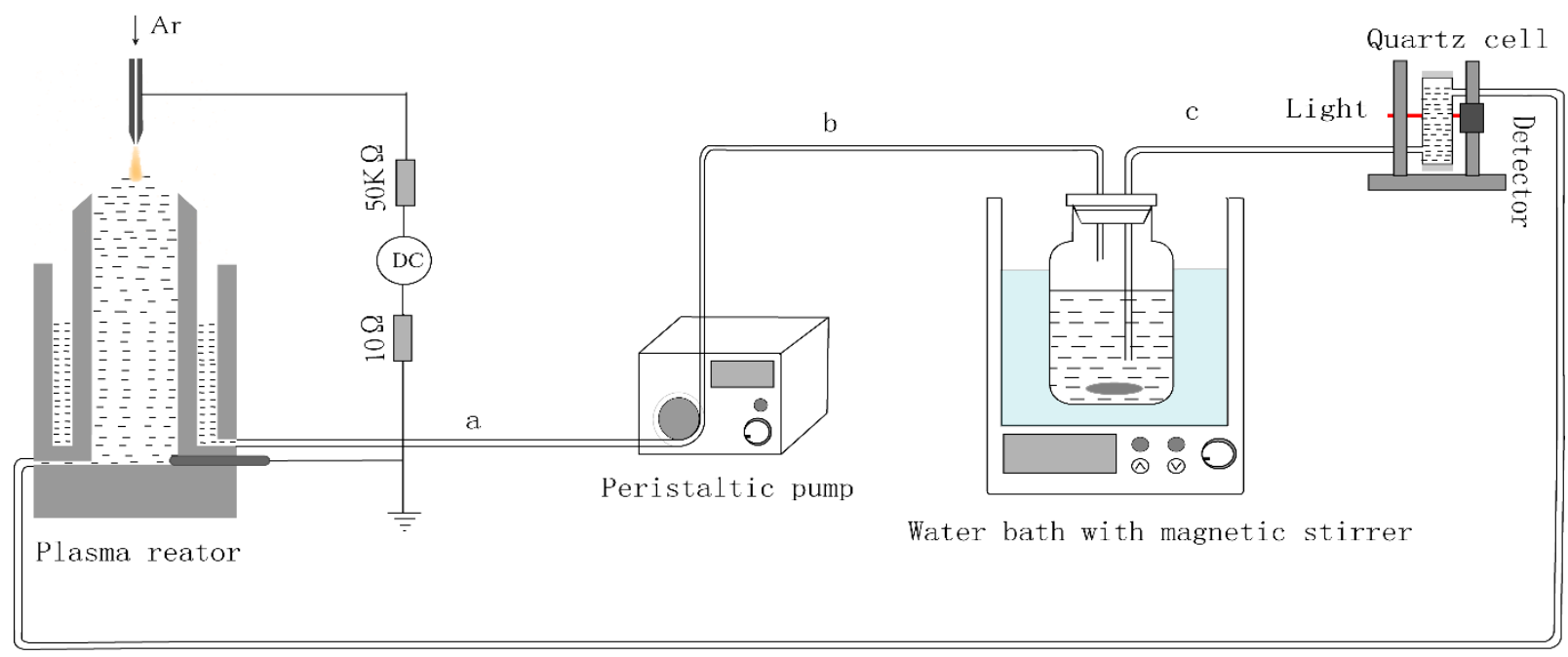

Fig1. Schematic diagram of the experimental set.

the reaction solution into a gas scrubber in a water bath and mixes with magnetic stirring (DFY-5L/10, Yu Hua, the water temperature is controlled at $25^{\circ} \mathrm{C}$ ) and then enter the cuvette (optical path length $10 \mathrm{~mm}$ ) to monitor the absorption spectrum of the solution in real time. Then the solution flows from the cuvette to the plasma circulating reactor, and the solution flowing from the bottom to the top of the reactor reacts with the plasma. Finally, the reacted solution returns to the peristaltic pump to enter the next cycle. The light source used in the experiment is a deuterium halide lamp (DH2000, Wenyi Optoelectronics); the spectrometer used for real-time detection of absorption spectra is (USB6500-Pro, Jingyi Optoelectronics).

The reagents used in the experiment are as follows: $\mathrm{AgNO}_{3}$ (analytical grade, Sinopharm Group); Polyvinyl Pyrrolidone $\left(\left(\mathrm{C}_{6} \mathrm{H}_{9} \mathrm{NO}\right)_{\mathrm{n}}\right.$, excellent grade pure, Sinopharm Group); isopropanol $\left(\mathrm{C}_{3} \mathrm{H}_{8} \mathrm{O}\right.$, analytical grade, Sinopharm Group) ), Glucose $\left(\mathrm{C}_{6} \mathrm{H}_{12} \mathrm{O}_{6}\right.$, analytical pure, Sinopharm Group). The ratio of $\mathrm{AgNO}_{3}$ to PVP concentration used in the experiment is $1: 2$. The ratio of $\mathrm{AgNO}_{3}$ to Glucose concentration used in the experiment is also 1:2.

\section{Results}

The $\mathrm{OH}$ radicals generated in the reaction system will directly or indirectly affect the reactivity of the solution and the synthesis process of Ag-NPs. Figure 2(a) is a graph of the plasmon resonance absorption intensity of the $\mathrm{AgNO}_{3} / \mathrm{PVP}$ mixed solution discharged for 20 minutes. It is found that the peak of the solution is around $400 \mathrm{~nm}$, indicating that the spherical silver nanoparticles have been successfully synthesized. Compared with Figure 2(a), it can be found from Figure 2(b) that the $\mathrm{OH}$ radical scavenger isopropanol has a great influence on the LSPR of $\mathrm{Ag}$ nanoparticles. Although $\mathrm{Ag}$ nanoparticles have a resonance absorption peak at $400 \mathrm{~nm}$. However, the intensity of the local plasmon resonance absorption peak is significantly enhanced compared with not using isopropanol. It can be seen from Figure 2(c) that as the concentration of isopropanol added increases, the reduction reaction becomes more intense, and the intensity of the absorption peak increases more obviously.

As the reaction progresses, the hydroxide radicals in the solution will indirectly generate hydrogen peroxide through direct passage. The oxidation reaction and the reduction reaction in the solution will proceed simultaneously, so there is a competitive relationship between the two reactions. The oxidizing substance in the solution is mainly hydrogen peroxide, and the electrons generated during the reaction of isopropanol with the discharge plasma will quickly react with $\mathrm{OH}$ radicals, so isopropanol can scavenge $\mathrm{OH}$ radicals. As the content of $\mathrm{OH}$ radicals in the solution decreases, the concentration of hydrogen peroxide also decreases. Therefore, the oxidation reaction in the solution is suppressed and the reduction reaction is promoted.

As the concentration of isopropanol increases, the rate of reduction increases. But when the reduction rate is too fast, the concentration of silver ions in the solution will be consumed in a short time. Therefore, the absorption peak intensity will begin to flatten and no longer increase.

(a)
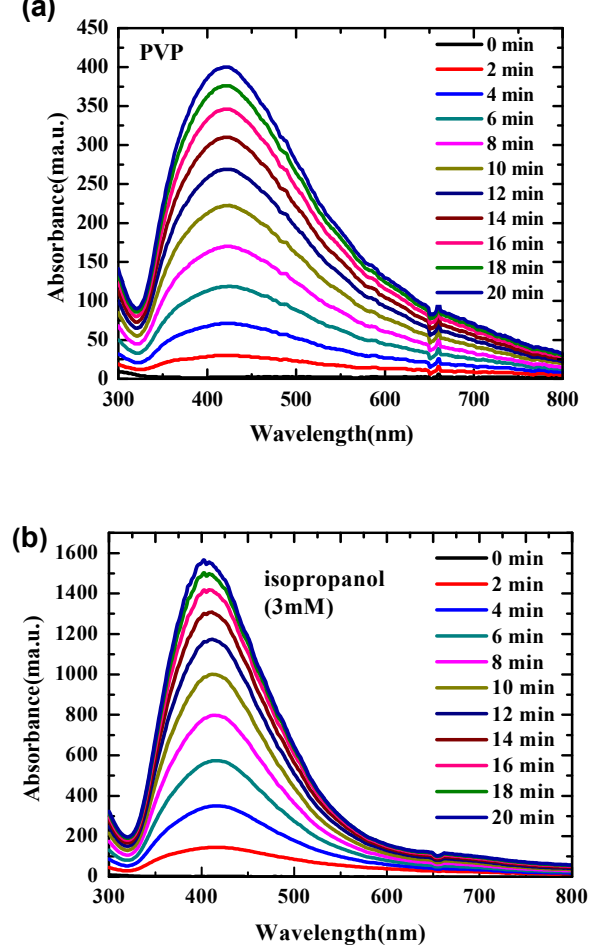

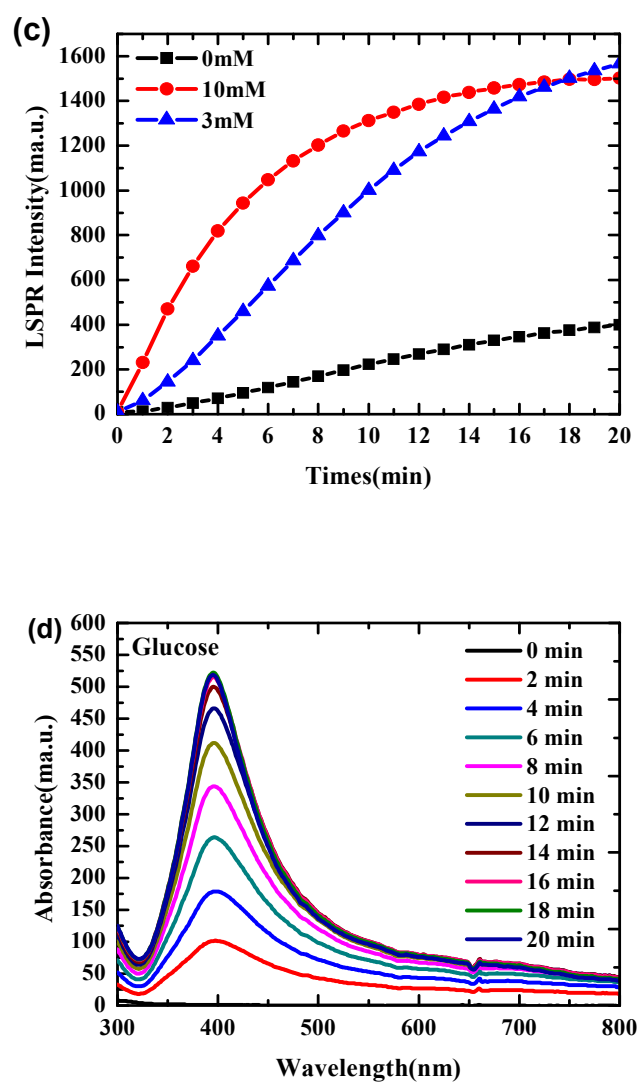

Fig2. (a) Time dependence of LSPR on Ag nanoparticles synthesized from $\mathrm{AgNO}_{3} / \mathrm{PVP}$ solution,(b) Time dependence of LSPR on Ag nanoparticles synthesized from $\mathrm{AgNO}_{3} / \mathrm{PVP} /$ isopropanol solution,(c) The dependence of the absorption peak intensity on Ag nanoparticles synthesized from different concentrations of isopropanol solution,(d) Time dependence of LSPR on $\mathrm{Ag}$ nanoparticles synthesized from $\mathrm{AgNO}_{3} /$ glucose solution.

The reducibility of glucose will change with the difference of $\mathrm{PH}$. In the plasma-liquid reaction system, the $\mathrm{pH}$ of the solution is less than 8 , so the reducibility of glucose is weak ${ }^{[11]}$. From the comparison of Figure2 (a) and (d), it is found that the peak position of the resonance absorption peak of the solution added with glucose has blue-shifted, and the half-width of the absorption peak is smaller.The absorption peak curve starts to overlap at 20 minutes of reaction, and the absorption peak intensity has reached saturation.

In the plasma-liquid reaction system, the $\mathrm{PH}$ of the solution is less than 8 . At this time, there are two main reactions that can reduce $\mathrm{Ag}^{+}$to $\mathrm{Ag}$ : one is that $\mathrm{e}_{\mathrm{aq}}{ }^{-}$and $\mathrm{H}$ react with $\mathrm{Ag}^{+}$; the other is that glucose directly reduces $\mathrm{Ag}^{+}$to $\mathrm{Ag}$. In the reaction system, glucose can directly react with $\mathrm{Ag}^{+}$to generate $\mathrm{Ag}$ elemental substance. Compared with the solution with PVP as a stabilizer, the introduction of glucose brings a new way to generate $\mathrm{Ag}$ for the reaction system, which greatly promotes Ag-NPs.

In our synthesis, glucose plays two roles: one is to reduce $\mathrm{Ag}^{+}$to $\mathrm{Ag}^{0}$, and the other is to act as a stabilizer for $\mathrm{Ag}$ atoms. In the process of reducing the metal salt to the corresponding metal nanoparticle, part of the glucose is oxidized to carboxylic acid. This carboxylic acid can produce a dense coating on the surface of Ag nanoparticles to counteract the van der Waals force responsible for particle coalescence, thereby stabilizing Ag nanoparticles [12]

In addition, the biomedical field requires high product safety. Glucose is a non-toxic reagent. Using glucose as a stabilizer can integrate the synthesized particles into various application systems. Glucose, as a small molecule stabilizer, can be easily removed when attached to the surface of nanoparticles. Therefore, the nanomaterials synthesized by this method have better biocompatibility.

\section{Conclusion}

Plasma-assisted synthesis of silver nanoparticles has the advantages of high efficiency and environmental protection, and the growth process of silver nanoparticles can be monitored in real time through local plasmon resonance absorption spectroscopy. It is found that the addition of isopropanol can greatly increase the absorption peak intensity of the solution and reduce its half-height width; greatly improve the synthesis efficiency and product uniformity. Compared with PVP, adding glucose has a dual effect, not only as a stabilizer, but also to promote the synthesis of silver nanoparticles. It has a more efficient synthesis effect, can complete the synthesis of nanoparticles in a short time, and its synthesis efficiency is significantly improved.

\section{Acknowledgments}

This work was supported by the doctoral scientific research fund of Guangxi Normal University (Grant No.2017BQ019).

\section{References}

1. Azharuddin M, Zhu G H, Das D, et al. A repertoire of biomedical applications of noble metal nanoparticles[J]. Chemical Communications, 2019,55:6964-6996

2. Kowalska E, Endo M, Wei Z, et al. Noble Metal Nanoparticles for Water Purification[M]//Nanoscale Materials in Water Purification. Elsevier, 2019: 553579.

3. Krysiak O A, Barczuk P J, Bienkowski K, et al. The photocatalytic activity of rutile and anatase $\mathrm{TiO} 2$ electrodes modified with plasmonic metal nanoparticles followed by photoelectrochemical measurements[J]. Catalysis Today, 2019, 321: 52-58.

4. Ağbulut Ü, Sarıdemir S. A general view to converting fossil fuels to cleaner energy source by adding nanoparticles[J]. International Journal of Ambient Energy, 2019: 1-6.

5. Wu J, Li F, Hu X, et al. Responsive Assembly of Silver Nanoclusters with a Biofilm Locally Amplified Bactericidal Effect to Enhance Treatments against Multi-Drug-Resistant Bacterial Infections[J]. ACS Central Science, 2019:1366-1376.

6. Xia Y, Xiong Y, Lim B, et al. Shape - controlled synthesis of metal nanocrystals: simple chemistry meets complex physics?[J]. Angewandte Chemie 
International Edition, 2009, 48(1): 60-103.

7. Tochikubo F, Shimokawa Y, Shirai N, et al. Chemical reactions in liquid induced by atmospheric-pressure dc glow discharge in contact with liquid[J]. Japanese Journal of Applied Physics, 2014, 53(12): 126201.

8. Chen Q, Li J, Li Y. A review of plasma-liquid interactions for nanomaterial synthesis[J]. Journal of Physics D: Applied Physics, 2015, 48(42): 424005.

9. Chen Q, Kaneko T, Hatakeyama R. Rapid synthesis of water-soluble gold nanoparticles with control of size and assembly using gas-liquid interfacial discharge plasma[J]. Chemical Physics Letters, 2012, 521: 113-117.

10. Gong X, Ma Y, Lin J, et al. Tuning the Formation Process of Silver Nanoparticles in a Plasma Electrochemical System by Additives[J]. Journal of The Electrochemical Society, 2018, 165(11): E540E545.

11. T. V. Bukreeva, I. V. Marchenko, B. V. Parakhonskiy et al. "Formation of silver nanoparticles on shells of polyelectrolyte capsules using silver-mirror reaction". Colloid Journal, 2009, 71(5): 596.

12. M. Giersig, T. Ung, L. M. Liz-Marzan et al. "ChemInform Abstract: Direct Observation of Chemical Reactions in Silica-Coated Gold and Silver Nanoparticles." Cheminform, 2010, 28(31): no-no. 